\title{
Characterization and genome annotation of a newly detected bacteriophage infecting multidrug-resistant Acinetobacter baumannii
}

\author{
Zichen Yang ${ }^{1,2} \cdot$ Xinzhu Liu $^{1} \cdot$ Yunlong Shi ${ }^{1} \cdot$ Supeng Yin ${ }^{1,2} \cdot$ Wei Shen $^{2} \cdot$ Jing Chen $^{1} \cdot$ Yu Chen $^{1} \cdot$ Yajie Chen $^{1} \cdot$ Bo You $^{1}$. \\ Yali Gong ${ }^{1} \cdot$ Xiaoqiang Luo $^{1} \cdot$ Cheng Zhang $^{1} \cdot$ Zhiqiang Yuan $^{1} \cdot$ Yizhi Peng $^{1}$
}

Received: 17 December 2018 / Accepted: 20 February 2019 / Published online: 21 March 2019

(c) The Author(s) 2019

\begin{abstract}
A novel virulent bacteriophage, $\varphi$ Abp2, infecting multidrug-resistant (MDR) Acinetobacter baumannii was isolated from the wastewater of a sewage management centre at Southwest Hospital, China. Transmission electron microscopy and phylogenetic analysis revealed that $\varphi$ Abp2 belongs to the subfamily Peduovirinae. A one-step growth curve demonstrated that $\varphi$ Abp 2 had a latent period of $15 \mathrm{~min}$, a lysis period of $35 \mathrm{~min}$, and a burst size of 222 particles per infected host cell. Moreover, $\varphi$ Abp2 showed a relatively broad host range in local A. baumannii, and it also exhibited tolerance over a wider range of thermal and $\mathrm{pH}$ conditions. Genomic sequencing revealed that $\varphi \mathrm{Abp} 2$ has a circular double-stranded DNA genome with no sequence similarity to our previously isolated $\varphi \mathrm{Abp} 1$. Eighty-eight putative open reading frames (ORFs) encoding 41 proteins of known function and 47 of unknown function were identified, and the G/C content was $37.84 \%$. $\varphi$ Abp2 is a new member of the subfamily Peduovirinae of the family Myoviridae. Its genome sequence is very similar to that of the $A$. baumannii phage LZ35.
\end{abstract}

\section{Introduction}

Acinetobacter baumannii is a growing threat that is responsible for numerous healthcare-associated infections, such as burn and wound infections [1]. It is among the leading causes of infections of the respiratory and urinary tracts, secondary meningitis, and sepsis [2]. Some strains of this widespread Gram-negative pathogen have been reported recently to be resistant to nearly all known antibiotics, and there is therefore an urgent need to find alternative treatments for these infections $[3,4]$.

Handling Editor: T. K. Frey.

Electronic supplementary material The online version of this article (https://doi.org/10.1007/s00705-019-04213-0) contains supplementary material, which is available to authorized users.

Yizhi Peng

yizhipengtmmu@163.com

1 State Key Laboratory of Trauma, Burns and Combined Injury, Institute of Burn Research, Southwest Hospital, Chongqing, China

2 Department of microbiology, College of Basic Medicin, Third Military Medical University (Army Medical University), Chongqing, China
In the middle of the 1910s, it was suggested that bacteriophages could be used successfully for treatment of human infections [5]. In recent decades, several reports have revealed the existence of multidrug-resistant (MDR) A. baumannii strains with a high degree of resistance to $\beta$-lactam antibiotics, including cephalosporins and penicillins [6]. To combat MDR A. baumannii isolates, phages are now being considered as alternatives to antibiotics, a century after their discovery [7]. In recent decades, there has been increasing evidence of the feasibility of using phage therapy to treat drug-resistant bacterial infections [8,9]. Indeed, not only can active phage be applied directly in the clinic [10], but new potential phage-derived antimicrobial agents are being identified and certified [11]. Thus, bacteriophage therapy is a potential strategy to fight MDR A. baumannii. However, problems including narrow host range, unclear hostphage interactions, and safety issues largely limit its clinical application.

Recently, we performed a national study of MDR A. baumannii epidemiology in which a number of bacteriophages were screened and preserved. A novel phage with strong lytic efficiency in MDR A. baumannii was isolated from wastewater from the intensive care unit of a burn treatment centre in southwestern China. Sequence analysis showed that this phage was completely different from our previous 
reported A. baumannii $\varphi A b p 1$ [12], and it was therefore named $\varphi A b p 2$. In this article, we describe the characterization and genome annotation of the phage $\varphi \mathrm{Abp} 2$, which will provide important information for its further study and application.

\section{Materials and methods}

\section{Bacterial preparations}

All of the MDR A. baumannii strains were collected previously for an epidemiology study and stored at the Institute of Burn Research, Southwest Hospital, China. MDR A. baumannii cultures were inoculated at a dilution of 1:100 and grown aerobically overnight at $37^{\circ} \mathrm{C}$ in LB (Luria-Bertani) broth or on LB solid medium.

\section{Antibiotic susceptibility tests (ASTs)}

Antibiotic susceptibility tests of clinically important bacteria and fungi were performed and interpreted according to the criteria of the Clinical and Laboratory Standards Institute [13] for the corresponding year, and the manufacturers' instructions were followed for the use of antibiotics.

To assess the resistance of pathogens to other antibiotics, the K-B disk diffusion method was applied. Nine antibiotics were selected: piperacillin, cefoperazone/sulbactam, sulfamethoxazole, ceftazidime, cefotaxime, imipenem, gentamicin, ciprofloxacin, and tigecycline (Oxoid, UK). Polymyxin B was not included in antibiotic susceptibility tests because a preliminary test showed no resistance among the collected strains (Table S1).

\section{Phage isolation and preparation}

A conventional screening method was used for bacteriophage isolation [14]. A sewage water sample (500-1000 ml) that had not been disinfected was collected from the sewage management centre of Southwest Hospital. MDR A. baumannii was then cultured from roughly sterilized sewage, and the culture was filtered through a $0.22-\mu \mathrm{m}$ membrane to collect bacteriophages, after which the sample was centrifuged at $13000 \times g$ for $15 \mathrm{~min}$ at room temperature. The double-layer agar method was applied to identify target bacteriophages [14]. Briefly, $10 \mu \mathrm{l}$ of supernatant and $100 \mu \mathrm{l}$ of a MDR A. baumannii strain were combined for a 10-minute absorption period and then mixed with $3 \mathrm{ml}$ of melted $0.7 \%$ soft agar. The mixture was then plated on an LB agar plate and incubated at $37{ }^{\circ} \mathrm{C}$ overnight. Clear plaques were picked aseptically and suspended in $500 \mu \mathrm{l}$ of liquid
LB medium. The process was repeated three times to isolate the phage. A new single-clone lytic bacteriophage plaque was picked and resuspended at $180 \mathrm{rpm}$ in $6 \mathrm{ml}$ of TM solution (10 mM Tris-HCl, $1 \mathrm{mM}$ EDTA, [pH 8.0]) from the potentially inhomogeneous bacteriophage mixture. The SM supernatant containing the target bacteriophage was filtered through a $0.22-\mu \mathrm{m}$ membrane and stored at $4{ }^{\circ} \mathrm{C}$. The lysed strains were excluded from the next round of bacteriophage isolation to avoid spectrum overlap with previously isolated bacteriophages, as described previously [15].

To obtain $\varphi A b p 2$ particles on a large scale, single phage plaques were suspended and incubated overnight at $37{ }^{\circ} \mathrm{C}$ with shaking at $160 \mathrm{rpm}$. Then, DNase I and RNase A were added to the culture to a final concentration of $1 \mu \mathrm{g} / \mathrm{ml}$, and incubation was continued at $37{ }^{\circ} \mathrm{C}$ for $30 \mathrm{~min}$. $\mathrm{NaCl}$ was added at a concentration of $5.84 \mathrm{~g} / 100 \mathrm{ml}$, mixed and dissolved, and the sample was then immersed in an ice bath for $1 \mathrm{~h}$. The sample was centrifuged at $10000 \times g$ for $10 \mathrm{~min}$, and the supernatant was collected. Solid PEG 8000 (Oxoid, UK) was added to a final concentration of $10 \%(\mathrm{w} / \mathrm{v})$, mixed and dissolved, and the sample was incubated in an ice bath overnight to let the phage pellet form a precipitate, which was collected by centrifugation at $12000 \times g$ for $10 \mathrm{~min}$ at $4{ }^{\circ} \mathrm{C}$, and the supernatant was discarded. Next, $2 \mathrm{ml}$ per $100 \mathrm{ml}$ of the original bacterial solution was added, adding TM solution to suspend the precipitate. Then, an equal volume of chloroform was added to the TM solution with shaking for $30 \mathrm{~s}$, the sample was centrifuged $5000 \times g$ for $10 \mathrm{~min}$, and the upper aqueous phase was collected. The extract was again extracted once with an equal volume of chloroform to obtain crude granules of $\varphi \mathrm{Abp} 2$.

\section{Transmission electron microscopy (TEM)}

The purified phage suspension was dropped onto a copper grid surface and negatively stained with $2 \%$ uranyl acetate for approximately $15 \mathrm{~s}$, and the excess stain was removed immediately. To examine the morphology of $\varphi \mathrm{Abp} 2$, the copper grid was inspected under a $120-\mathrm{kV}$ transmission electron microscope (JEM-1400 Plus, JEOL LTD, Japan).

\section{Thermal and pH stability}

Following our previously described method [9], the stability (titre) of $\varphi \mathrm{Abp} 2$ under different thermal and $\mathrm{pH}$ conditions was tested. Briefly, the purified phage was incubated at different temperatures $\left(-80,-20,4,20,40,60\right.$ and $\left.80^{\circ} \mathrm{C}\right)$ for $48 \mathrm{~h}$ and at various $\mathrm{pH}$ levels (from 1 to 14 ) for $1 \mathrm{~h}$. Then, the double-layer agar method was used to determine the titre of the incubated bacteriophages every $1 \mathrm{~h}$. Both thermal and $\mathrm{pH}$ stability tests were performed in triplicate. 


\section{One-step growth curve}

For one-step growth experiments, MDR A. baumannii were infected with $\varphi \mathrm{Abp} 2$ at a multiplicity of infection (MOI) of 0.1 using a 10-min adsorption time. Samples were centrifuged at $13,000 \times g$ for $30 \mathrm{~s}$ to clear unabsorbed phages from the supernatants. The procedure was performed twice, using LB medium for washing. Then, the A. baumannii pellets with adsorbed $\varphi \mathrm{Abp} 2$ were resuspended in $4 \mathrm{ml}$ of LB liquid medium. After that, the cultures were grown at $37{ }^{\circ} \mathrm{C}$ with shaking at $160 \mathrm{rpm}$, during which samples were collected at 5- or 10-min intervals up to $100 \mathrm{~min}$. The number of $\varphi \mathrm{Abp} 2$ particles was immediately determined by the previously described double-layer agar method. This measurement was performed in triplicate.

\section{Host range determination and efficiency of plating}

The host range of $\varphi \mathrm{Abp} 2$ was determined using a spotting method as described previously [16]. In brief, 60 strains of MDR A. baumannii from different locations were included for host range testing (Table S2). All MDR A. baumannii strains were incubated overnight at $37{ }^{\circ} \mathrm{C}$ with $\varphi \mathrm{Abp} 2$ to allow cell lysis. Plaques were inspected for lytic activity based on their clarity and transparency. The host range determination was performed in triplicate.

A more thorough assessment of productive infection as defined by the efficiency of plating (EOP) was conducted. Each strain was tested three times with each of four different dilutions of $\varphi \mathrm{Abp} 2$ that had resulted in lysis in the host range determination. This was performed under the same conditions as in the spot assays. The phage lysates were diluted $10^{6}$ - to $10^{9}$-fold. The plates were incubated overnight at $30{ }^{\circ} \mathrm{C}$, and the number of plaque-forming units (PFU) was determined for each combination. When the $10^{6}$ dilution did not result in any plaques, a lower dilution was tried afterwards to verify that the EOP was lower than 0.001. Finally, the EOP was calculated (average PFU on target bacteria/ average PFU on host bacteria) along with the standard deviation for the three measurements.

\section{DNA extraction, sequencing and genome analysis}

Phage genomic DNA was extracted using the phenol-chloroform protocol [17]. Briefly, crude granules of $\varphi \mathrm{Abp} 2$ were lysed by the addition of proteinase $\mathrm{K}(100 \mathrm{mg} / \mathrm{ml})$ at $37{ }^{\circ} \mathrm{C}$ in water for $30 \mathrm{~min}$. Then, EDTA $(0.5 \mathrm{mM}, \mathrm{pH} 8.0)$ was added to stop the digestion of the $\varphi$ Abp2 sample. An equal volume of phenol was added to the mixture, which was then centrifuged at $5,000 \times g\left(4{ }^{\circ} \mathrm{C}\right)$ for $10 \mathrm{~min}$ to remove debris. This step was repeated for a second time with chloroform. Then, the supernatant was incubated overnight with isoamyl alcohol at $-20{ }^{\circ} \mathrm{C}$ to precipitate the DNA. After washing three times with cold $75 \%$ ethanol, the $\varphi$ Abp2' genomic DNA was dissolved in TE buffer, quantified using a NanoDrop spectrophotometer (Thermo Scientific, Waltham, MA, USA) and sequenced on an Illumina HiSeq 2500 sequencer (San Diego, CA, USA) with a $2 \times 100$-bp read length.

Through sequencing the cloned fragments of the $\varphi \mathrm{Abp} 2$ genome, more than 7,300-fold coverage was obtained. Then, the short reads were assembled using SOAPdenovo (http:// sourceforge.net/projects/soapdenovo2/fles/SOAPdenovo $2 /$ ) to the genome sequence. The assembled whole genome sequence was searched against the current nucleotide databases (http://www.ncbi.nlm.nih.gov/) using the Basic Local Alignment Search Tool (BLAST). Putative protein-encoding open reading frames (ORFs) were jointly predicted using online bioinformatic tools, including GeneMark (http://topaz .gatech.edu/GeneMark/), FgenesB (http://linux1.softberry. $\mathrm{com} /$ berry.phtml?topic $=$ fgenesb \& group $=$ programs\&subgr oup=gfindb), Rast (http://rast.nmpdr.org/) and Glimmer (http://ccb.jhu.edu/software/glimmer/index.shtml). Then, the intersecting predicted ORFs from different databases and algorithms were examined manually. Protein BLAST (BLASTP) (http://www.ncbi.nlm.nih.gov/BLAST/) was applied to match putative proteins sharing similarities with the predicted phage ORFs. Phylogenetic analysis of phage large terminase subunit sequences was performed using ClustalW (https://www.ebi.ac.uk/Tools/msa/clustalw2/) and MEGA 6 (https://www.megasoftware.net/). The annotated $\varphi$ Abp2 genome sequence was submitted to the NCBI database through Sequin (https://www.ncbi.nlm.nih.gov/Sequi n/) under accession number MF346584.1.

\section{Results}

\section{Morphology of the phage $\varphi$ Abp2}

$\varphi$ Abp2 targeting an MDR A. baumannii strain was screened from the sewage management centre at Southwest Hospital. According to the TEM image of Abp2, the isometric polyhedral head was $85.5 \pm 3.4 \mathrm{~nm}$ in diameter, whereas the contractile tail was $86.4 \pm 3.4 \mathrm{~nm}$ in length and $23.3 \pm 2.4 \mathrm{~nm}$ in width (Supplementary Fig S1). Thus, $\varphi$ Abp2 was designated to the family Myoviridae, order Caudovirales, following the current guidelines of the ICTV (International Committee on Taxonomy of Viruses, http://ictv.global/taxonomyRelease s.asp).

\section{Life cycle of phage $\varphi$ Abp2}

The life cycle of $\varphi \mathrm{Abp} 2$ was investigated by performing a one-step growth experiment. The duration of the latent period was short $\left(\sim 15 \mathrm{~min}\right.$ at $\left.37^{\circ} \mathrm{C}\right)$ and was followed by a lysis period that lasted $50 \mathrm{~min}$. The burst size was 222 
phage particles per infected cell after $100 \mathrm{~min}$ (Supplementary Fig S2). Moreover, $\varphi$ Abp2 was stable at temperatures ranging from -80 to $20^{\circ} \mathrm{C}$. However, the viral titres decreased slightly at $40{ }^{\circ} \mathrm{C}$ and were reduced dramatically at 60 and $80{ }^{\circ} \mathrm{C}$ (Supplementary Fig S3A). In addition, the $\mathrm{pH}$ tolerance of $\varphi \mathrm{Abp} 2$ was stable over a broad range (from 5.0-10.0). However, the titre dropped significantly at $\mathrm{pH} 4.0$ and pH 11.0 (Supplementary Fig S3B).

\section{Host range}

Sixty MDR A. baumannii strains from different locations were selected for a phage host-range test to determine the lytic range of phage $\varphi A b p 2$. All of the local MDR A. baumannii strains tested were vulnerable to phage $\varphi$ Abp2 (Table 1), while none of the allopatric strains were lysed except for one strain from XiAn and three strains from Nanjing (Supplementary Table S2), indicating that $\varphi \mathrm{Abp} 2$ is capable of lysing local strains but is limited to a specific geographic area.

\section{Genome annotation and analysis}

High-throughput sequencing reads were assembled into a completely closed, circular genome sequence using SOAPdenovo. The circularity of the phage genome was confirmed by restriction endonuclease mapping (data not shown). This double-stranded DNA genome of $\varphi$ Abp 2 consisted of 45,373 bp, with a GC content of $37.84 \%$ (Fig. 1). BLASTn analysis of the whole genome sequence revealed that the genome of $\varphi \mathrm{Abp} 2$ shared $0 \%$ sequence identity with our previously isolated $\varphi \mathrm{Abp} 1$, suggesting that they are unrelated to each other. However, the $\varphi \mathrm{Abp} 2$ genome sequence exhibited 93\% nucleotide sequence identity, with $71 \%$ coverage to $A$. baumannii phage LZ35, with a number of ORFs nevertheless differing significantly (Table S3). The genome was found to contain 88 putative ORFs (Fig. 1). ORFs encoding proteins with known functions were classified into several groups: those associated with morphogenesis and structure, DNA replication, repair, recombination and processing, biological metabolism, transcription, lysis, assembly and
Table 1 Host range summary of phage AbP2

\begin{tabular}{llcl}
\hline Bacterial source & Number lytic (n/10) & Percentage lytic & EOP $^{\wedge}$ \\
\hline BICU*, Southwest Hospital, Chongqign & $10 / 10$ & $100 \%$ & 1.0 \\
XiAn & $2 / 10$ & $20 \%$ & 0.3 \\
He Nan province & $0 / 10$ & $0 \%$ & N/A $^{\#}$ \\
BICU, Honghui Hospital, Shenzhen & $0 / 10$ & $0 \%$ & N/A \\
Ji Lin province & $0 / 10$ & $0 \%$ & N/A \\
Nanjing & $4 / 10$ & $40 \%$ & 0.5 \\
\hline
\end{tabular}

*BICU, burn intensive care unit

${ }^{\wedge} \mathrm{EOP}$, average efficiency of plating

\#N/A not tested; phage AbP2 did not lyse the bacterium

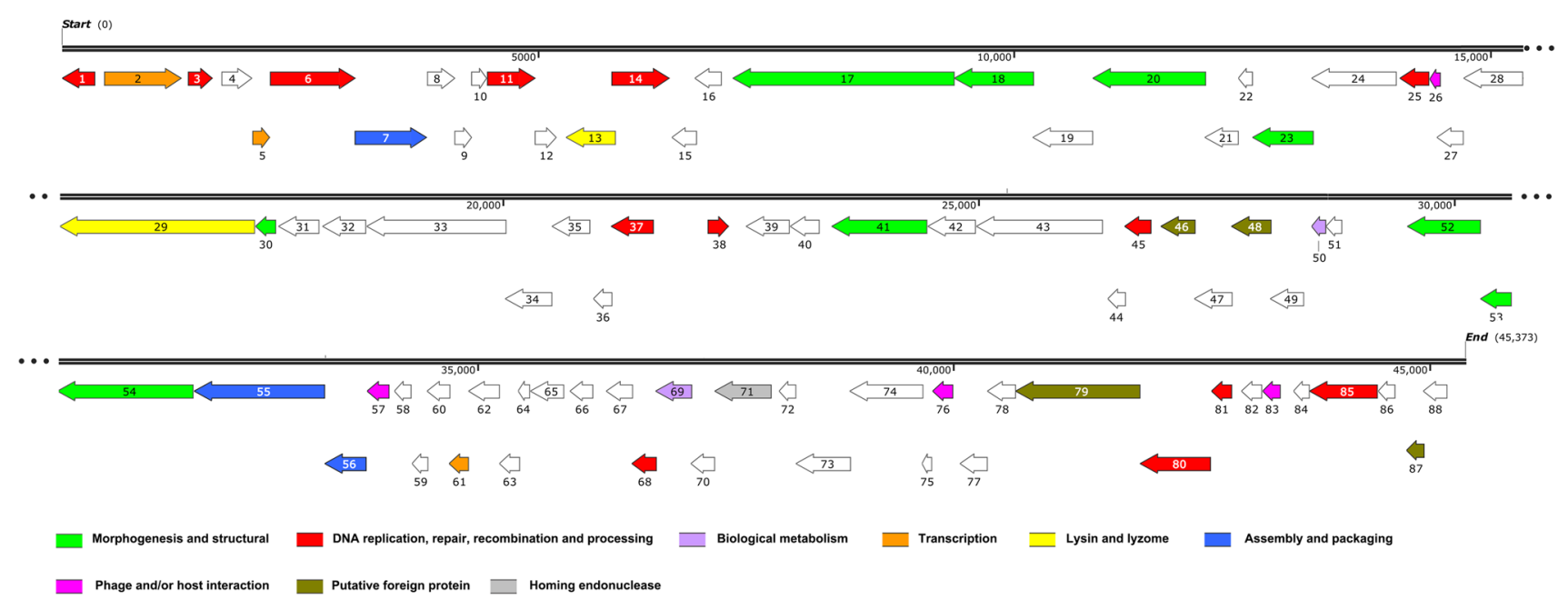

Fig. 1 Detailed $\varphi$ Abp2 genome annotation. Predicted genes are represented by arrows with their direction of transcription indicated by the direction of each arrow. Different colours indicate different functional groups of genes 
packaging, phage and/or host interaction, putative foreign proteins, and homing endonucleases (Fig. 1).

Structure- and assembly-associated proteins identified in BLASTn and BLASTp analysis include the tail fibre protein (ORF17, 18 and possibly ORF30), baseplateassociated protein (ORF20 and 23), putative capsid protein (ORF41), head protein (ORF 52) and portal protein (ORF54). Genes encoding DNA replication, repair, recombination and processing modules, including transposase (ORF1 and 68), GTPase (ORF3), recombinase (ORF6), nucleoside triphosphate pyrophosphohydrolase (ORF11), nuclease (ORF14), methyltransferase (ORF38 and 45), helicase (ORF79), primosomal protein (ORF81), and DNA-binding protein (ORF85), were distributed throughout the whole $\varphi$ Abp2 phage genome (Fig. 2, Table S3). Among these sequences, 41 ORFs encoded proteins with high levels of similarity to phage proteins with similar functions, while 47 undefined ORFs were also identified. We identified ORFs with biological metabolism, transcription, lysin and lyzome, assembly and packaging, phage and/or host interaction, and homing endonuclease functions (Fig. 1), but four ORFs (ORF46, 48, 80, and 87) were predicted to encode putative foreign proteins, although the level of sequence identity was only $24-42 \%$ (Table S3).

\section{Phylogenetic analysis}

To further classify $\varphi \mathrm{Abp} 2$, we aligned the amino acid sequence of the terminase large subunit proteins (encoded by ORF55) with those from other phages of the family Myoviridae, using MEGA6 software. A neighbour-joining tree generated from the alignments revealed that $\varphi \mathrm{Abp} 2$ clustered with Peduovirinae phages such as bacteriophage 186 and Aeromonas phage phiO18P (Fig. 2). The characteristics of the phylogenetic tree and its unique genome features indicate that $\varphi \mathrm{Abp} 2$ is a new member of the family Myoviridae, order Caudovirales.

\section{Discussion}

In this study, we present the characterization and genomic annotation of $\varphi$ Abp2, a lytic phage from a clinical MDR $A$. baumannii isolate with strong lytic ability and a wider host range than those of our previously screened bacteriophages [14].

TEM and phylogenetic analysis indicated that phage $\varphi$ Abp 2 should be assigned to the subfamily Peduovirinae based on its morphological characteristics and its phylogenetic relationship to phages of the family Myoviridae. Based on its morphology and genome features, phage $\varphi$ Abp2 was determined to be a new member of the subfamily Peduovirinae of the family Myoviridae, order Caudovirales.

One-step growth curve analysis revealed a 15-min latent period, a 35-min lysis period and a burst size of 222 phage particles per infected host cell, which is smaller than that of $\varphi$ Abp1 [14]. Compared with our previously isolated $\varphi$ Abp1, $\varphi \mathrm{Abp} 2$ had a smaller burst size [14] but a wider range of $\mathrm{pH}$ and thermal tolerance [9] as well as a broader host range
Fig. 2 Phylogenetic analysis of the $\varphi A b p 2$ large terminase subunit (ORF55). The amino acid sequences of the large terminase subunits were compared using MEGA6, and a phylogenetic tree was generated by the neighbour-joining method with 1000 bootstrap replicates

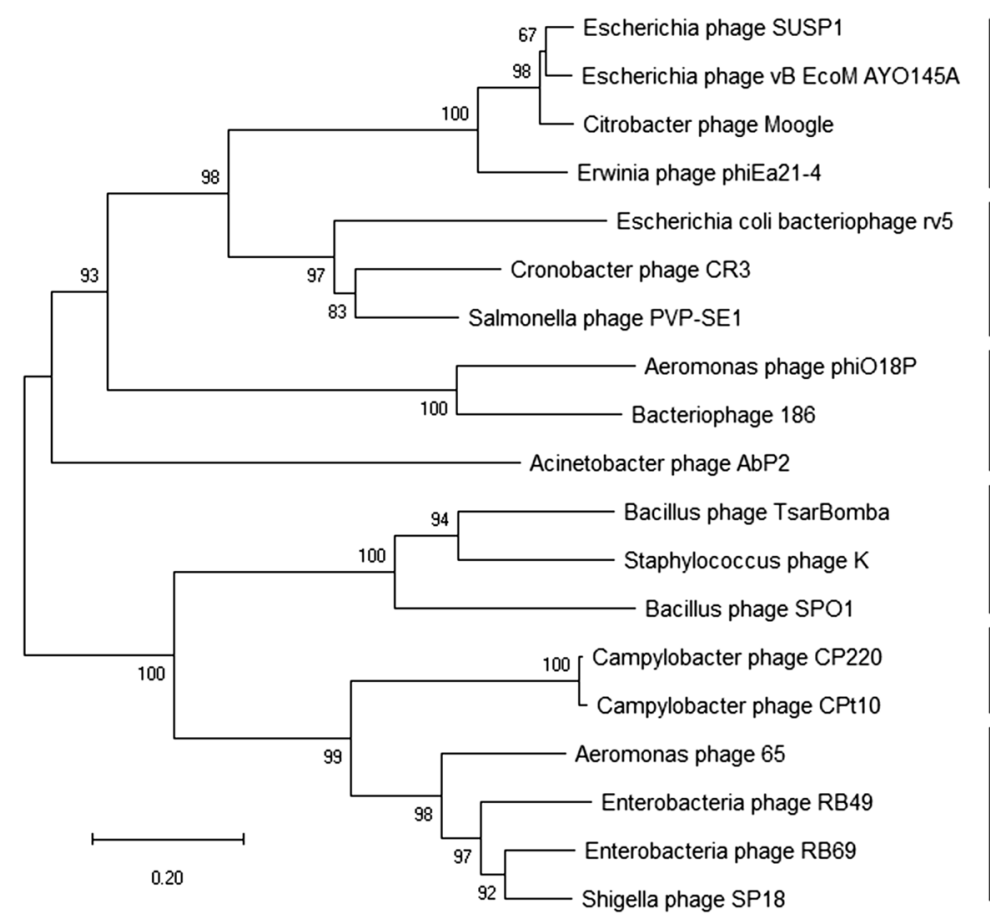

Ounavirinae

Ounavirinae

Vequintavirinae

Peduovirinae

Spouavirinae

Eucampyvirinae

Tevenvirinae 
among local MDR A. baumannii strains, making $\varphi$ Abp2 a more suitable candidate than $\varphi A b p 2$ for clinical application.

The high mutation rate of bacteriophages makes it difficult to identify gene functions [18], and this lack of information limits their application and causes uncertainty regarding their safety $[19,20]$. There are many similarities between the genes of $\varphi \mathrm{Abp} 2$ and those of previously identified bacteriophages. The genome sequence of phage $\varphi \mathrm{Abp} 2$ showed the highest nucleotide sequence identity to that of A. baumannii phage LZ35. Functional prediction revealed 88 possible ORFs, of which only 41 showed relatively high amino acid sequence similarity to other hypothetical proteins. The other 47 (53.4\%) ORFs could not be annotated, indicating that $\varphi A b p 2$ is a unique phage. Moreover, the lack of clarity about the function of many of the genes creates obstacles to broadening the narrow host spectrum of this bacteriophage. Previous studies have identified host-range determinants such as gp17 in T7 phage and gp37 and gp3 in T4-like phages [21,22], but none of the currently known host range determinants showed similarity to the ORFs of $\varphi A b p 2$, suggesting that the unannotated ORFs might hold the key to the specific host range characteristics of $\varphi$ Abp2.

The putative foreign genes of $\varphi$ Abp2 (ORF46, 48, 80, and 87 ) showed a degree of similarity to genes of nonmicrobial organisms suggesting that this phage can carry foreign genes and possibly even human genes. It is proposed that $\varphi$ Abp2 may interact directly or indirectly with these organisms, since phages might acquire foreign genes to allow them to produce soluble factors [23]. To determine whether phage $\varphi$ Abp 2 might be temperate, we searched for lysogenic phage genes, but found only one possible lysogenic gene (ORF30). Although no known lysogenic factors, including excisionase or anti-repressor proteins [24], were found during the genome annotation of $\varphi \mathrm{Abp} 2$, the predicted proteins with unknown functions might nevertheless have lysogen-related functions. The presence of ORFs (ORF46 and 80) with similarity to two genes of Homo sapiens suggests that these genes might have been acquired from humans. The application of $\varphi \mathrm{Abp} 2$ to humans might lead to complications, including immune effects, as phages have been shown recently to interact directly with somatic cells [25-27]. The presence of foreign gene fragments reminds us that the clinical use of phages will require caution, and it might be more suitable to use "clean, simple" engineered phages in clinical practice.

\section{Conclusion}

We isolated and sequenced the genome of phage $\varphi \mathrm{Abp} 2$, which has a broad host lysis spectrum in native strains and broad tolerance, which improves its prospects for clinical application. Its host spectrum requires further research at the genetic level. A putative endolysin gene (ORF13) was identified in the phage $\varphi$ Abp2 genome. Future work should examine potential applications of $\varphi \mathrm{Abp} 2$ in the treatment of MDR $A$. baumannii and the interaction between $\varphi \mathrm{Abp} 2$ and its hosts.

Open Access This article is distributed under the terms of the Creative Commons Attribution 4.0 International License (http://creativeco mmons.org/licenses/by/4.0/), which permits unrestricted use, distribution, and reproduction in any medium, provided you give appropriate credit to the original author(s) and the source, provide a link to the Creative Commons license, and indicate if changes were made.

\section{References}

1. Howard A, O'Donoghue M, Feeney A, Sleator RD (2012) Acinetobacter baumannii: an emerging opportunistic pathogen. Virulence. 3(3):243-250

2. Peleg AY, Seifert H, Paterson DL (2008) Acinetobacter baumannii: emergence of a successful pathogen. Clin Microbiol Rev 21(3):538-582

3. Gong Y, Shen X, Huang G, Zhang C, Luo X, Yin S et al (2016) Epidemiology and resistance features of Acinetobacter baumannii isolates from the ward environment and patients in the burn ICU of a Chinese hospital. J Microbiol (Seoul, Korea). 54(8):551-558

4. Huang G, Yin S, Gong Y, Zhao X, Zou L, Jiang B et al (2016) Multilocus sequence typing analysis of carbapenem-resistant Acinetobacter baumannii in a Chinese Burns Institute. Front Microbiol. 7:1717

5. Roach DR, Debarbieux L (2017) Phage therapy: awakening a sleeping giant. Emerg Top Life Sci. 1(1):93-103

6. Hsu LY, Apisarnthanarak A, Khan E, Suwantarat N, Ghafur A, Tambyah PA (2017) Carbapenem-Resistant Acinetobacter baumannii and Enterobacteriaceae in South and Southeast Asia. Clin Microbiol Rev 30(1):1-22

7. Sharma S, Chatterjee S, Datta S, Prasad R, Dubey D, Prasad RK et al (2017) Bacteriophages and its applications: an overview. Folia Microbiol 62(1):17-55

8. Huang G, Shen X, Gong Y, Dong Z, Zhao X, Shen W et al (2014) Antibacterial properties of Acinetobacter baumannii phage Abp1 endolysin (PlyAB1). BMC Infect Dis 14:681

9. Yin S, Huang G, Zhang Y, Jiang B, Yang Z, Dong Z et al (2017) Phage Abp1 rescues human cells and mice from infection by pan-drug resistant Acinetobacter baumannii. Cell Physiol Biochem 44(6):2337-2345

10. Schooley RT, Biswas B, Gill JJ, Hernandez-Morales A, Lancaster J, Lessor L et al (2017) Development and use of personalized bacteriophage-based therapeutic cocktails to treat a patient with a disseminated resistant Acinetobacter baumannii infection. Antimicrob Agents Chemother. 61(10):e00954

11. Brown ED (2004) Drugs against superbugs: private lessons from bacteriophages. Trends Biotechnol 22(9):434-436

12. Zhang Z, Schwartz S, Wagner L, Miller W (2000) A greedy algorithm for aligning DNA sequences. J Comput Biol 7(1-2):203-214

13. CLSI (2012) M100-S25 Performance standards for antimicrobial susceptibility testing. Clinical and Laboratory Standards Institute, Wayne

14. Huang G, Le S, Peng Y, Zhao Y, Yin S, Zhang L et al (2013) Characterization and genome sequencing of phage Abp1, a new phiKMV-like virus infecting multidrug-resistant Acinetobacter baumannii. Curr Microbiol 66(6):535-543 
15. Mattila S, Ruotsalainen P, Jalasvuori M (2015) On-demand isolation of bacteriophages against drug-resistant bacteria for personalized phage therapy. Front Microbiol. 6:1271

16. Ceyssens PJ, Minakhin L, Van den Bossche A, Yakunina M, Klimuk E, Blasdel B et al (2014) Development of giant bacteriophage varphiKZ is independent of the host transcription apparatus. J Virol 88(18):10501-10510

17. Castro-Mejia JL, Muhammed MK, Kot W, Neve H, Franz CM, Hansen LH et al (2015) Optimizing protocols for extraction of bacteriophages prior to metagenomic analyses of phage communities in the human gut. Microbiome. 3:64

18. Harrison E, Brockhurst MA (2017) Ecological and evolutionary benefits of temperate phage: what does or doesn't kill you makes you stronger. BioEssays. 39(12):1700112

19. Tiwari R, Chakraborty S, Dhama K, Wani MY, Kumar A, Kapoor S (2014) Wonder world of phages: potential biocontrol agents safeguarding biosphere and health of animals and humans- current scenario and perspectives. PJBS. 17(3):316-328

20. Orndorff PE (2016) Use of bacteriophage to target bacterial surface structures required for virulence: a systematic search for antibiotic alternatives. Curr Genet 62(4):753-757

21. Chen M, Zhang L, Abdelgader SA, Yu L, Xu J, Yao H et al (2017) Alterations in gp37 expand the host range of a T4-like phage. Appl Environ Microbiol 83(23):e01576-17
22. Trojet SN, Caumont-Sarcos A, Perrody E, Comeau AM, Krisch HM (2011) The gp38 adhesins of the T4 superfamily: a complex modular determinant of the phage's host specificity. Genome Biol Evol. 3:674-686

23. Abedon ST, Lejeune JT (2007) Why bacteriophage encode exotoxins and other virulence factors. Evol Bioinform Online. 1:97-110

24. Fogg PCM, Rigden DJ, Saunders JR, McCarthy AJ, Allison HE (2011) Characterization of the relationship between integrase, excisionase and antirepressor activities associated with a superinfecting Shiga toxin encoding bacteriophage. Nucleic Acids Res 39(6):2116-2129

25. Nguyen S, Baker K, Padman BS, Patwa R, Dunstan RA, Weston TA et al (2017) Bacteriophage transcytosis provides a mechanism to cross epithelial cell layers. mBio 8(6):e01874-17

26. Barr JJ (2017) A bacteriophages journey through the human body. Immunol Rev 279(1):106-122

27. Gorski A, Dabrowska K, Miedzybrodzki R, Weber-Dabrowska B, Lusiak-Szelachowska M, Jonczyk-Matysiak E et al (2017) Phages and immunomodulation. Future Microbiol. 12:905-914

Publisher's Note Springer Nature remains neutral with regard to jurisdictional claims in published maps and institutional affiliations. 